OPEN ACCESS

Edited by:

Ding Ding,

Fudan University, China

Reviewed by:

Janet Mifsud

University of Malta, Malta

Qianyi Xiao

Fudan University, China

${ }^{*}$ Correspondence:

Qing Zhang

nxzhangqing@aliyun.com

Specialty section:

This article was submitted to

Neuroepidemiology,

a section of the journal

Frontiers in Neurology

Received: 26 September 2018 Accepted: 03 January 2019 Published: 29 January 2019

Citation:

Wang X, Chao L, Liu X, XU X and Zhang Q (2019) Association Between

HLA Genotype and Cutaneous Adverse Reactions to Antiepileptic

Drugs Among Epilepsy Patients in Northwest China. Front. Neurol. 10:1. doi: 10.3389/fneur.2019.00001

\section{Association Between HLA Genotype and Cutaneous Adverse Reactions to Antiepileptic Drugs Among Epilepsy Patients in Northwest China}

\author{
Xu Wang ${ }^{1}$, Lina Chao ${ }^{2}$, Xiaojing Liu $^{3}$, Xianrui X ${ }^{1}$ and Qing Zhang ${ }^{1,4 *}$ \\ ${ }^{1}$ Department of Neurology, General Hospital of Ningxia Medical University, Yinchuan, China, ${ }^{2}$ Department of Neurology, The \\ First People's Hospital, Shizuishan, China, ${ }^{3}$ Department of Neurology, The First Hospital of Tongxiang, Tongxiang, China, \\ ${ }^{4}$ Ningxia Key Laboratory of Cerebrocranial Diseases, The Incubation Base of National Key Laboratory, Yinchuan, China
}

This study aimed to investigate the association between HLA genotypes and antiepileptic drug-induced cutaneous adverse reactions (AEDs-cADRs) among patients with epilepsy in Ningxia Hui Autonomous Region of Northwest China. Fifteen patients with AEDs-CADRs and 30 matched AEDs tolerant controls from anested case-control study were tested the HLA-A, HLA-B, and HLA-DRB1 genotype using the polymerase chain reaction sequence-based typing (PCR-SBT). Significant difference was not observed between AEDs-CADRs and AEDs tolerant groups in terms of HLA-A, HLA-B, and HLA-DRB1 genotype frequencies. Future studies using larger cohorts are needed to verify this observation.

Keywords: antiepileptic drugs, Chinese, epilepsy, HLA genotype, cutaneous adverse reaction

\section{INTRODUCTION}

Cutaneous adverse drug reactions (cADRs) are common adverse reactions observed in patients using antiepileptic drugs (AEDs). Studies have demonstrated that the incidence of AEDs-cADRs was about $3.61 \%$. Also, cADRs are relatively common with the use of aromatic antiepileptic drugs (AAEDs), including carbamazepine (CBZ), phenytoin (PHT), lamotrigine (LTG), and phenobarbital (PB) (1). They manifest as ordinary maculopapular eruption (MPE), eventually leading to serious life-threatening conditions such as hypersensitivity syndrome (HSS), Steven-Johnson syndrome (SJS), or toxic epidermal necrolysis (TEN). AEDs-cADRs often lead to drug discontinuation in patients with epilepsy, resulting in the inability to control seizures. A $40 \%$ mortality rate has been reported in patients with severe cADRs (2).

Since 2004, a number of studies have suggested a strong association of HLA genotype with the occurrence of AEDs-cADRs. However, this association differs between different races and areas (3-5). In China, the majority of studies have been carried out in southern Chinese Han population (6-8). Since the seventh century, central Asians, Arabs, and Persians have migrated to China and settled to gradually form the Hui ethnicity. Some studies have suggested genetic differences between the Hui and the Han (9). Ningxia, located in Northwest China, has the largest Hui population in China. Therefore, Ningxia is an ideal state to study regional and ethnic differences. A nested case-control study was conducted in patients who were AEDs-cADRs and AEDs tolerant to determine the association between HLA genotypes and patients with AEDs-cADRs in Ningxia. 


\section{MATERIALS AND METHODS}

\section{Study Participants}

Study participants were patients diagnosed with epilepsy by the Department of Neurology in Ningxia Medical University General Hospital. The inclusion criteria were as follows: (1) Ningxia resident with no history of marriages with other ethnic groups for more than three generations; (2) Clear indications for AEDs treatment; (3) Have not been administered oral AEDs, and potential adverse drug reactions declared in patients or their guardians, after which signed informed consents were obtained; and (4) The initial dose and increasing dose of AEDs determined according to the "Pharmacopeia of People's Republic of China" (2010 edition). The exclusion criteria were as follows: (1) Having a history of alcohol-related epilepsy; (2) Having a treatable cause (such as metabolic disorders, poisoning, and infection); (3) With progressive brain or central nervous system diseases, such as encephalitis, tumors, or degenerative diseases; (4) Suffering from other diseases and the emergence of allergy during the follow-up period; and (5) Having to discontinue or substitute medications and not completing 12 weeks of prescribed oral AEDs.

Four hundred and fifteen patients were followed up bi-weekly for 12 weeks after initiating oral AEDs. The initial dosage of PHT, LTG, CBZ, and valproate (VPA) was 200, 500, 12.5, 100 $\mathrm{mg} / \mathrm{d}$, and $5 \mathrm{mg} / \mathrm{kg} / \mathrm{d}$, respectively. They were examined for symptoms and signs of cADRs in an epileptic clinic every 2 weeks. AEDs tolerance was defined as patients who were able to tolerate AEDs without cADRs manifestation. If cADRs manifested, the AEDs were discontinued immediately and a dermatologist was consulted to diagnose and treat the patients (Figure 1).

Two attending or one chief physician from the Department of Dermatology examined the patients. The criteria for the diagnosis and classification of cADRs were as follows: (1) MPE: a rash, not involving the mucosa, no organ or system damage, and resolved after 1-2 weeks; (2) HSS: in addition to skin rash, numerous viscera involvement with systemic manifestations, such as fever, arthralgia, eosinophilia, and lymphadenopathy; (3) SJS: the occurrence of skin exfoliation, involving a range of no
$<10 \%$ of the body area, with or without other organ or system damage; (4) TEN: the presence of skin exfoliation, involving more than $30 \%$ of the body area, with or without other organ or system damage; and (5) SJS/TEN: the presence of skin exfoliation, involving a range of $10-30 \%$ of the total body area. The patients were treated for skin damage based on the severity as determined by a dermatologist after cADRs diagnosis was confirmed. These patients were assigned to the AEDs-cADRs group.

Nested case-control design is the most common way to reduce the costs of exposure assessment in prospective epidemiological studies. They can also reduce the sample size through matching (10). In this study, 15 patients with epilepsy who developed cADRs were defined as the AEDs-cADRs group. For each patient with AEDs-cADRs, two patients with AEDs tolerance were selected and matched by AEDs, gender, age ( \pm 3 years), and ethnicity.

\section{Clinical Data Collection}

A unified AEDs-cADRs epidemiological questionnaire, including demographics, underlying diseases, medication history, allergies, and seizure history, was used. The occurrence of cADRs during the 12 weeks was recorded, including the date of cADRs manifestation and other clinical manifestations involving the mucosa and subtypes.

\section{Ethics Statement}

The General Hospital of Ningxia Medical University Ethics Committee approved the study. Also, the study was performed in compliance with the Helsinki Declaration. Access to the patient information database was granted by the General Hospital of Ningxia Medical University and approved by the ethics committee following study review. All enrolled patients agreed to have their data published and signed a written informed consent form.

HLA-A, HLA-B, and HLA-DRB1 Genotyping

Peripheral venous blood $(3 \mathrm{~mL})$ from each participant was collected in anticoagulant tubes. An extraction kit (Beijing

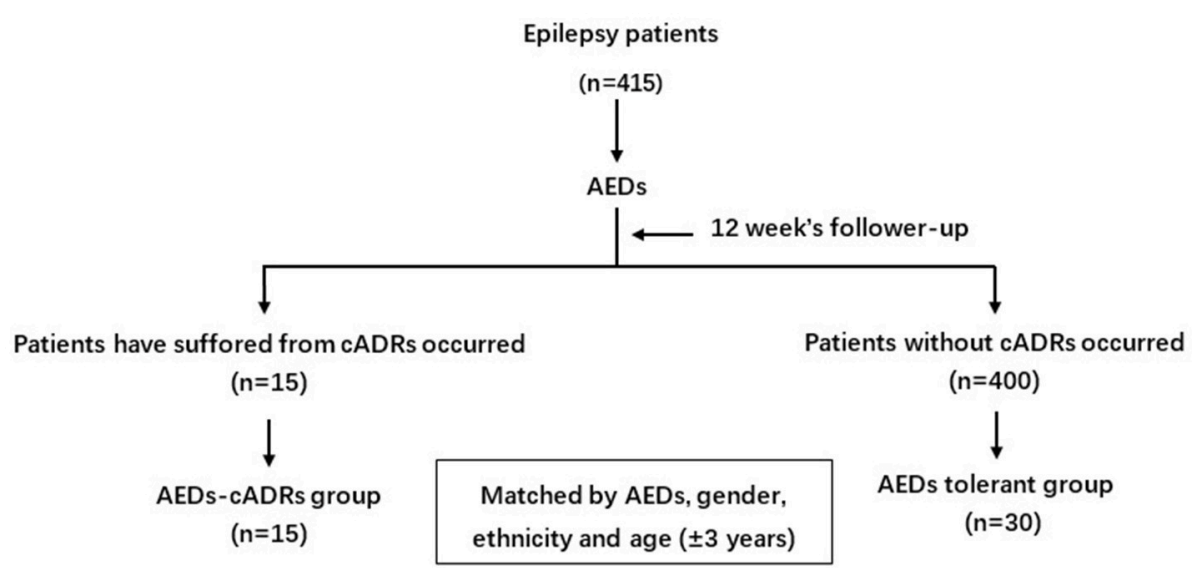

FIGURE 1 | Flow chart of the nested case-control study. 
TABLE 1 | The characteristics of AEDs-cADRs group.

\begin{tabular}{|c|c|c|c|c|c|c|c|c|c|}
\hline $\begin{array}{l}\text { AEDs-cADRs } \\
\text { group }\end{array}$ & Gender & $\begin{array}{c}\text { Age } \\
\text { (years) }\end{array}$ & Ethnicity & Address (city) & Allergy history & Type of AEDs & Daily dose & $\begin{array}{c}\text { Time of } \\
\text { occurrence }\end{array}$ & Type of cARDs \\
\hline $\mathrm{C} 01$ & $M$ & 19 & Han & Zhongning & No & PHT & 300 mg & After $6 \mathrm{~m}$ & MPE \\
\hline C02 & $M$ & 14 & Han & Shizuishan & No & LEV & $1250 \mathrm{mg}$ & After $5 \mathrm{~d}$ & MPE \\
\hline C03 & $\mathrm{F}$ & 20 & Han & Yinchuan & No & $\mathrm{CBZ}$ & 100 mg & After $6 \mathrm{~d}$ & MPE \\
\hline $\mathrm{CO} 4$ & $M$ & 41 & Han & Yinchuan & No & $\mathrm{CBZ}$ & 200 mg & After $43 d$ & MPE \\
\hline C05 & $\mathrm{F}$ & 25 & Han & ShiZuiShan & No & LTG & $6.25 \mathrm{mg}$ & After $5 \mathrm{~d}$ & MPE \\
\hline C06 & $\mathrm{F}$ & 31 & Hui & Yinchuan & YES & LTG & $50 \mathrm{mg}$ & After $7 \mathrm{~d}$ & MPE \\
\hline C07 & $\mathrm{M}$ & 14 & Han & ShiZuiShan & No & LEV & 500 mg & After $1 \mathrm{~m}$ & MPE \\
\hline C08 & $\mathrm{M}$ & 27 & Han & ShiZuiShan & No & LTG & $12.5 \mathrm{mg}$ & After $2 \mathrm{~m}$ & MPE \\
\hline C09 & $\mathrm{F}$ & 41 & Hui & Pengyang & No & LTG & $25 \mathrm{mg}$ & After $1 \mathrm{~m}$ & HSS \\
\hline C10 & $M$ & 55 & Han & Yinchuan & No & LTG & $25 \mathrm{mg}$ & After $2 \mathrm{~m}$ & MPE \\
\hline C11 & $M$ & 40 & Hui & Yongning & No & LTG & $50 \mathrm{mg}$ & After $15 d$ & MPE \\
\hline C12 & $M$ & 22 & Han & Jingyuan & No & LTG & $25 \mathrm{mg}$ & After $13 d$ & MPE \\
\hline C13 & $M$ & 60 & Han & ZhongNing & No & VPA & 400 mg & After $1 \mathrm{~d}$ & MPE \\
\hline C14 & $\mathrm{F}$ & 16 & Han & Guyuan & No & LTG & $12.5 \mathrm{mg}$ & After $10 \mathrm{~d}$ & MPE \\
\hline C15 & $M$ & 55 & Han & Yinchuan & No & LTG & $25 \mathrm{mg}$ & After $5 \mathrm{~d}$ & MPE \\
\hline
\end{tabular}

TABLE 2 | The characteristics of AEDs tolerant group.

\begin{tabular}{|c|c|c|c|c|c|c|c|c|c|c|c|}
\hline $\begin{array}{l}\text { AEDs tolerant } \\
\text { group }\end{array}$ & Gender & $\begin{array}{c}\text { Age } \\
\text { (years) }\end{array}$ & Ethnicity & Address (city) & $\begin{array}{l}\text { Type of } \\
\text { AEDs }\end{array}$ & $\begin{array}{l}\text { AEDs } \\
\text { tolerance group }\end{array}$ & $\begin{array}{l}\text { Address } \\
\text { (city) }\end{array}$ & Gender & $\begin{array}{c}\text { Age } \\
\text { (years) }\end{array}$ & Ethnicity & Type of AEDs \\
\hline M01 & $\mathrm{M}$ & 24 & Han & Qingtongxia & PHT & M16 & Yongning & $\mathrm{M}$ & 26 & Han & LTG \\
\hline M02 & M & 24 & Han & Yongning & PHT & M17 & Yongning & $\mathrm{F}$ & 25 & Hui & LTG \\
\hline M03 & M & 17 & Han & Jingyuan & LEV & M18 & Yongning & $\mathrm{F}$ & 25 & Hui & LTG \\
\hline M04 & M & 24 & Han & Yanchi & LEV & M19 & Yinchuan & M & 30 & Han & LTG \\
\hline M05 & $\mathrm{F}$ & 27 & Han & Helan & CBZ & M20 & Yinchuan & $\mathrm{M}$ & 65 & Han & LTG \\
\hline M06 & $\mathrm{F}$ & 25 & Han & Zhongwei & CBZ & M21 & Helan & $\mathrm{M}$ & 35 & Han & LTG \\
\hline M07 & M & 45 & Han & Wuzhong & CBZ & M22 & Helan & M & 36 & Han & LTG \\
\hline M08 & M & 39 & Han & Zhongning & CBZ & M23 & Shizuishan & $M$ & 17 & Han & LTG \\
\hline M09 & $\mathrm{F}$ & 17 & Han & Yingchuan & LTG & M24 & Zhongwei & $\mathrm{M}$ & 28 & Han & LTG \\
\hline M10 & $\mathrm{F}$ & 22 & Han & Yongning & LTG & M25 & Zhongwei & $\mathrm{M}$ & 32 & Han & VPA \\
\hline M11 & $\mathrm{F}$ & 27 & Hui & Yongning & LTG & M26 & Yinchuan & $\mathrm{M}$ & 12 & Han & VPA \\
\hline M12 & $\mathrm{F}$ & 20 & Hui & Yanchi & LTG & M27 & Qingtongxia & $\mathrm{F}$ & 20 & Han & LTG \\
\hline M13 & $\mathrm{F}$ & 23 & Han & Yinchuan & LEV & M28 & Helan & $\mathrm{F}$ & 44 & Han & LTG \\
\hline M14 & $\mathrm{M}$ & 21 & Han & Yinchuan & LEV & M29 & Helan & $\mathrm{M}$ & 23 & Han & LTG \\
\hline M15 & $\mathrm{M}$ & 35 & Han & Helan & LTG & M30 & Zhongning & $\mathrm{M}$ & 30 & Han & LTG \\
\hline
\end{tabular}

Tiangen Biotech Company, China) was used to extract genomic DNA from whole blood according to the manufacturer's protocols. HLA genotype was performed using PCR-SBT at the Beijing Boao Crystal Biotechnology Company, China. The following procedural steps were adopted: (1) amplification of HLA-A, HLA-B, and HLA-DRB1 loci; (2) purification and detection of the amplified products; and (3) HLA genotyping sequencing using a $3730 \mathrm{XL}$ ABI detector.

\section{Statistical Analysis}

Continuous variables were expressed as mean and standard deviation (SD) and categorical variables as frequencies (\%). The Pearson chi-squared test was used to compare categorical variables and the Student $t$-test to compare continuous variables. Differences in HLA genotype frequency between the groups were analyzed using the Fisher's exact test. Risk association between HLA alleles and AEDs-cADRs were presented as odds ratios (OR) and 95\% confidence intervals (CI). P-values and 95\% CIs were estimated using two-tailed tests. Data were analyzed using SPSS13.0 software.

\section{RESULTS}

\section{Characteristics of the Study Participants}

The age of the 15 patients with AEDs-cARDs ranged from 14 to 60 years old,with an average age of $39.2 \pm 15.4$ years old. These included 10 male patients and 5 female patients with a ratio of 2:1. Twelve patients were of Han ethnicity and three of Hui ethnicity. Ten patients were with generalized epileptic seizures and five were with partial epileptic seizures. One patient 
TABLE 3 | Genotypes of HLA-A, HLA-B, and HLA-DRB1 in AEDs-CADRs group.



In the HLA naming protocol, "**" separates the HLA locus (A, B and DRB1) from the serotype (32, 33 and 35), eg. HLA-A* 3201.

TABLE 4 | Genotypes of HLA-A, HLA-B, and HLA-DRB1 in AEDs tolerant group.

\begin{tabular}{|c|c|c|c|c|c|c|c|}
\hline \multirow{2}{*}{$\begin{array}{l}\text { AEDs tolerant group } \\
\text { M01 }\end{array}$} & \multirow{2}{*}{$\begin{array}{l}\text { Type of AEDs } \\
\text { PHT }\end{array}$} & \multicolumn{6}{|c|}{ HLA sub-type } \\
\hline & & 1101 & 2601 & 0801 & 4001 & 0301 & 0405 \\
\hline M02 & PHT & 1101 & 1101 & 3503 & 5102 & 1101 & 1123 \\
\hline M03 & LEV & 1101 & 2402 & 0702 & 5401 & 0405 & 1501 \\
\hline M04 & LEV & 1101 & 1101 & 0702 & 4001 & 0101 & 0901 \\
\hline M07 & CBZ & 0302 & 1101 & 0801 & 1502 & 0301 & 1202 \\
\hline M08 & CBZ & 0201 & 3303 & 1501 & 5001 & 0701 & 1101 \\
\hline M09 & LTG & 0207 & 1101 & 0702 & 5101 & 0901 & 1501 \\
\hline M10 & LTG & 0201 & 3004 & 5801 & 5801 & 0410 & 1454 \\
\hline M11 & LTG & 1101 & 3001 & 1302 & 5201 & 0701 & 1502 \\
\hline M12 & LTG & 0201 & 3303 & 1301 & 5801 & 0301 & 1202 \\
\hline M16 & LTG & 0101 & 0101 & 4001 & 4001 & 1454 & 1454 \\
\hline M17 & LTG & 2402 & 2402 & 3802 & 5101 & 1201 & 1312 \\
\hline M18 & LTG & 2402 & 3001 & 3503 & 5401 & 1101 & 1501 \\
\hline M19 & LTG & 0203 & 3303 & 1801 & 5502 & 1104 & 1602 \\
\hline M20 & LTG & 0301 & 3303 & 5201 & 5801 & 0301 & 1502 \\
\hline M21 & LTG & 0201 & 2402 & 1501 & 5101 & 0901 & 1001 \\
\hline M22 & LTG & 1101 & 3101 & 0702 & 4001 & 0101 & 0901 \\
\hline M23 & LTG & 0201 & 2601 & 4006 & 4006 & 0803 & 0901 \\
\hline M24 & LTG & 3001 & 3101 & 1302 & 5101 & 0701 & 1454 \\
\hline M25 & VPA & 0201 & 0201 & 4006 & 4601 & 0901 & 1210 \\
\hline M26 & VPA & 0201 & 2601 & 1301 & 5701 & 0701 & 1202 \\
\hline M27 & LTG & 0201 & 2402 & 4801 & 5502 & 0901 & 1405 \\
\hline
\end{tabular}

In the HLA naming protocol, "**" separates the HLA locus (A, B and DRB1) from the serotype (32, 33 and 35), eg. HLA-A* 3201. 
had a pollen allergy history. One patient received PHT that induced CADRs, two patients received CBZ, two received LEV, one received VPA, and nine received LTG. Moreover, there were 14 patients with MPE and one Hui female patient with HSS who accepted treatment after onset. Further, five patients had cADRs after taking the initial dose of AEDs, of which one patient received $\mathrm{CBZ}$, one received $\mathrm{LEV}$, and three received LTG. The average time from patients taking AEDs to the occurrence of cADRs was $93.4 \pm 70$ days, with the longest latency period being 6 months and the shortest being 1 day (Table 1 ).

The age of the 30 AEDs tolerant patients ranged from 12 to 65 years old, with an average age of $31.7 \pm 12.4$ years old. These included 19 male patients and 11 female patients at a ratio of 1.7:1. Further, 26 patients were of Han ethnicity and 4 were of Hui ethnicity. Twenty-three had generalized seizures, and seven had partial seizures. Moreover, two received PHT, four received $\mathrm{LEV}$, four received CBZ, two received VPA, and eighteen received LTG (Table 2).

\section{Genotypes of HLA-A, HLA-B, and HLA-DRB1}

In the AEDs-cADRs group, the number of HLA-A, HLA$\mathrm{B}$, and HLA-DRB1 genotypes detected was 12, 18, and 15, respectively. Higher distribution frequencies of HLA-A genotype were $A^{*} 0207$ (16.67\%) and $A^{*} 2402$ (13.33\%). The highest distribution frequency of HLA-B genotype was B*5101 (20\%). The distribution frequency of $A^{*} 0201, A^{*} 0206, A^{*} 3101, A^{*} 3303$, B*3501, DRB1*0701, DRB1*0803, DRB1*0901, DRB1*1101, DRB1*1202, and DRB1*1454 was $10 \%$ (Table 3).

In the AEDs tolerant group, the number of HLA-A, HLA-B, and HLA-DRB1 genotypes detected was 13, 26, and 23 , respectively. Higher distribution frequencies of the HLAA genotype were $A^{*} 0201(21.67 \%)$ and $A^{*} 1101$ (20\%). The distribution frequency of the HLA-B genotype was lower than $10 \%$. Higher distribution frequency of the HLA-DRB1 genotype was $\mathrm{DRB}^{*} 0901$ (15\%), followed by $\mathrm{DRB1}{ }^{*} 0301(10 \%)$ and DRB1*1202 (10\%) (Table 4).

\section{Association Between HLA-A, HLA-B, and HLA-DRB1 Genotypes and AEDs-cADRs, LTG-CADRs, and AAEDs-cADRs}

As shown in Table 5, OR values of 15 HLA genotypes were $>1$. No significant differences in HLA genotype frequencies were observed between the AEDs-cADRs and AEDs tolerant group $(P$ $>0.05)$.

HLA genotyping in 9 patients with LTG-cADRs identified a total of 11 HLA-A types, 13 HLA-B types, and 13 HLA-DRB1 types. In 18 patients of LTG tolerant group, 12 HLA-A types,

TABLE 5 | Association between HLA-A, HLA-B, HLA-DRB1, and AEDs-cADRs.

\begin{tabular}{|c|c|c|c|c|}
\hline \multirow[t]{2}{*}{ HLA type } & \multicolumn{2}{|c|}{ Frequency } & \multirow[t]{2}{*}{ OR(95\%Cl) } & \multirow[t]{2}{*}{$P$-value } \\
\hline & $\begin{array}{l}\text { AEDs-cADRs group } \\
\qquad(2 n=30)\end{array}$ & $\begin{array}{l}\text { AEDs tolerant group } \\
\qquad(2 n=60)\end{array}$ & & \\
\hline$A^{\star} 0203$ & 1/30 (3.3\%) & $1 / 60(1.7 \%)$ & $2.034(0.12 \sim 33.70)$ & 1.00 \\
\hline$A^{\star} 0207$ & $5 / 30(16.7 \%)$ & $4 / 60(6.7 \%)$ & $2.800(0.69 \sim 11.32)$ & 0.15 \\
\hline$A^{*} 2402$ & 4/30 (13.3\%) & $7 / 60$ (11.7\%) & $1.165(0.31 \sim 4.34)$ & 1.00 \\
\hline$A^{*} 3101$ & $3 / 30$ (10.0\%) & $3 / 60(5.0 \%)$ & $2.111(0.40 \sim 11.15)$ & 1.00 \\
\hline$A^{*} 3303$ & $3 / 30$ (10.0\%) & $5 / 60(8.3 \%)$ & $1.222(0.27 \sim 5.50)$ & 1.00 \\
\hline$B^{\star} 1501$ & $2 / 30(6.7 \%)$ & 2/60 (3.3\%) & $2.071(0.28 \sim 15.48)$ & 0.60 \\
\hline$B^{*} 1502$ & 0/30 (0.0\%) & $1 / 60(1.7 \%)$ & $1.017(0.98 \sim 1.05)$ & 1.00 \\
\hline$B^{\star} 4002$ & $1 / 30$ (3.3\%) & 1/60 (1.7\%) & $2.034(0.12 \sim 33.70)$ & 1.00 \\
\hline$B^{\star} 4801$ & 1/30 (3.3\%) & $1 / 60$ (1.7\%) & $2.034(0.12 \sim 33.70)$ & 1.00 \\
\hline$B * 5102$ & 1/30 (3.3\%) & $1 / 60(1.7 \%)$ & $2.034(0.12 \sim 33.70)$ & 1.00 \\
\hline DRB1*0405 & 2/30 (6.7\%) & 2/60 (3.3\%) & $2.071(0.28 \sim 15.48)$ & 0.60 \\
\hline DRB1*0803 & $3 / 30$ (10.0\%) & $3 / 60$ (5.0\%) & $2.111(0.40 \sim 11.15)$ & 0.40 \\
\hline DRB1*1201 & 1/30 (3.3\%) & 1/60 (1.7\%) & $2.034(0.12 \sim 33.70)$ & 1.00 \\
\hline DRB1*1210 & 1/30 (3.3\%) & $1 / 60(1.7 \%)$ & $2.034(0.12 \sim 33.70)$ & 1.00 \\
\hline DRB1*1302 & 1/30 (3.3\%) & $1 / 60(1.7 \%)$ & $2.034(0.12 \sim 33.70)$ & 1.00 \\
\hline
\end{tabular}

TABLE 6 | Association between HLA-A, HLA-B, HLA-DRB1, and LTG-cADRs.

\begin{tabular}{|c|c|c|c|c|}
\hline \multirow[t]{2}{*}{ HLA type } & \multicolumn{2}{|c|}{ Frequency } & \multirow[t]{2}{*}{ OR(95\%Cl) } & \multirow[t]{2}{*}{$P$-value } \\
\hline & $\begin{array}{l}\text { LTG-cADRs group } \\
(2 n=18)\end{array}$ & $\begin{array}{l}\text { LTG tolerant group } \\
\qquad(2 n=36)\end{array}$ & & \\
\hline$A^{*} 0206$ & 4/18 (22.2\%) & 4/36 (11.1\%) & $2.28(0.50 \sim 10.5)$ & 0.29 \\
\hline$A^{*} 2402$ & 2/18 (11.1\%) & 2/36 (5.6\%) & $2.12(0.27 \sim 16.5)$ & 0.48 \\
\hline$A * 3101$ & 2/18 (11.1\%) & 2/36 (5.6\%) & $2.12(0.27 \sim 16.5)$ & 0.48 \\
\hline$B^{\star} 5101$ & 4/18 (22.2\%) & 2/36 (5.6\%) & 4.85 (0.80 29.6) & 0.76 \\
\hline DRB1*3303 & 3/18 (16.7\%) & 2/36 (5.6\%) & $3.40(0.51 \sim 22.5)$ & 0.19 \\
\hline
\end{tabular}


17 HLA-B types, and 17 HLA-DRB1 types were identified. OR values of 5 HLA genotypes were $>1$. No significant differences in HLA genotype frequency were found between the LTG-cADRs and LTGtolerant group $(P>0.05)$ (Table 6).

HLA genotyping in 12 patients with AAEDs-cADRs identified a total of 12 HLA-A types, 15 HLA-B types, and 15 HLADRB1 types. In 24 patients of AAEDs tolerant group, 13 HLA-A types, 23 HLA-B types, and 20 HLA-DRB1 types were identified. The OR values of 13 HLA genotypes were $>1$. No significant difference in HLA genotype frequency was observed between AAEDs-cADRs and AAEDs tolerant group $(P>0.05)$ (Table 7).

\section{DISCUSSION}

The occurrence of AEDs-cADRs in patients with epilepsy may be influenced by gender, age, initial AEDs dosage, incremental AEDs dosage, and adding rate, with or without a history of allergies, monotherapy or polytherapy, functional status of the liver and kidney, and genetic factors (11). Chung and colleagues reported that HLA-B* 1502 was strongly correlated with CBZ-SJS/TEN in Taiwan Han populations (4). This result was later supported by others (3), especially for serious cADRs, with HLA susceptibility genes being the most important factor.

Different AEDs induce different cADR symptoms. In a largescale study on 3,793 patients with epilepsy taking AEDs, the overall incidence rate of AEDs-cADRs reached 3.61\%. The incidence rates of AEDs induced by cADRs were as follows: LTG (11.11\%), OXC (8.92\%), CBZ (3.80\%), PHT (1.98\%), PB (0.42\%), VPA (0.57\%), and LEV (1.65\%) (1). About $88.41 \%$ of cADRs were induced by AAEDs of CBZ (47.56\%), LTG (17.07\%), OXC (9.15\%), PHT(9.15\%), and $\mathrm{PB}(5.49 \%)$ (12). These results suggested that AAEDs were more likely to induce cADRs in clinical practice compared with other types of AEDs. In the present study, $80 \%$ of patients with AEDs-cADRs were AAEDscADRs (12/15). Of these, LTG-cADRs was the most common $(9 / 15)$.
Correlation studies on HLA genotypes and AEDs-cADRs have been conducted in mainland China (6-8), Taiwan (4), Hong Kong (13), Southeast Asia (14-16), Japan (17), Korea (18), Europe (19), North America (20), and other regions. The reported correlations between HLA genotypes and AEDscADRs have the following characteristics: (1) Susceptible genes associated with AEDs-cADRs may be different among different races. For example, HLA-B*1502 is the susceptible gene for AEDs-SJS/TEN in Han Chinese and Southeast Asians. However, in Japan, Europe, and other parts of the world, the susceptible gene for CBZ-cADRs is HLA-A*3101. (2) In the Han population in Southeast Asia, HLA-B*1502 may have a susceptibility to aromatic AEDs-SJS/TEN. (3) The incidence rate of AEDscADRs is relevant to the distribution rate of HLA-B*1502 alleles among different races. The higher the distribution rate of HLA-B* 1502 in the race, the higher the incidence rates for AEDs-cADRs.

Ningxia, located in Northwest China, is an agglomeration of Hui ethnicities that are unlike the southern Han Chinese population genetically. Whether any HLA susceptibility genes are responsible for the occurrence of AEDs-cADRs among the northwestern population in China is not known. Therefore, the distribution rate of HLA genotypes was compared in the following groups: all patients in the AEDs-cADRs group vs. patients in the AEDs tolerant group, AAEDs-cADRs group vs. AAEDs tolerant group, and LTG-cADRs group vs. LTGtolerant group. The results suggested that the HLA-A, HLA-B, and HLADRB1 genotype distribution frequencies were not statistically significantly different between the two groups.

The present study had some limitations that might have impacted the outcome. First, selecting a large number of patients with AEDs-cADRs was difficult. Nine patients with LTG-cADRs were enrolled in this study. However, only one patient with CBZ-cADRs, one patient with PHT-cADRs, two patients with CBZ-cADRs, two patients with LEV-cADRs, and one patient with VPA-cADRs. Future studies focusing

TABLE 7 | Association between HLA-A, HLA-B, HLA-DRB1, and AAEDs-cADRs.

\begin{tabular}{|c|c|c|c|c|}
\hline HLA type & \multicolumn{2}{|c|}{ Frequency } & OR(95\%Cl) & $P$-value \\
\hline$A^{*} 0203$ & $1 / 24(4.2 \%)$ & $1 / 48(2.1 \%)$ & $2.043(0.12 \sim 34.16)$ & 1.00 \\
\hline$A^{*} 0207$ & 4/24 (16.7\%) & 4/48 (8.3\%) & 2.00 (0.50 9.70) & 0.42 \\
\hline$B^{\star} 1302$ & 2/24 (8.3\%) & $2 / 48(4.2 \%)$ & $2.091(0.28 \sim 15.83)$ & 0.60 \\
\hline$B^{*} 4801$ & $1 / 24(4.2 \%)$ & $1 / 48(2.1 \%)$ & $2.043(0.12 \sim 34.16)$ & 1.00 \\
\hline$B^{\star} 5101$ & 6/24 (25.0\%) & $5 / 48(10.4 \%)$ & $2.87(0.77 \sim 10.60)$ & 0.16 \\
\hline DRB1 ${ }^{\star} 0101$ & $1 / 24(4.2 \%)$ & $1 / 48(2.1 \%)$ & $2.043(0.12 \sim 34.16)$ & 1.00 \\
\hline DRB1*0405 & 2/24 (8.3\%) & $1 / 48(2.1 \%)$ & 4.273 (0.37 49.68) & 0.25 \\
\hline DRB1*0701 & 3/24 (12.5\%) & $3 / 48$ (6.3\%) & $2.143(0.40 \sim 11.15)$ & 0.39 \\
\hline
\end{tabular}


on large-sample populations with similar epilepsy and AEDs should be conducted. Second, the study did not adjust for age, gender, and other possible confounding variables during statistical analysis due to the small sample size. This might have had a minor impact on the results because the patients and controls were matched using a nested case-control design.

\section{CONCLUSIONS}

The presentstudy did not find a significant association between any HLA genotypes and AEDs-cADRs in patients with epilepsy in Northwest China. Future studies using larger cohorts are needed to verify this observation.

\section{REFERENCES}

1. Wang XQ, Lang SY, Shi XB, Tian HJ, Wang RF, Yang F. Antiepileptic drug-induced skin reactions: a retrospective study and analysis in 3793 Chinese patients with epilepsy. Clin Neurol Neurosurg. (2012) 114:862-5. doi: 10.1016/j.clineuro.2012.01.019

2. Abood GJ, Nickoloff BJ, Gamelli RL. Treatment strategies in toxic epidermal necrolysis syndrome: where are we at? J Burn Care Res. (2008) 29:269-76. doi: 10.1097/BCR.0b013e31815f3658

3. Grover S, Kukreti R. HLA alleles and hypersensitivity to carbamazepine: an updated systematic review with meta-analysis. Pharmacogenet Genomics (2014) 24:94-112. doi: 10.1097/FPC.0000000000000021

4. Chung WH, Hung SI, Hong HS, Hsih MS, Yang LC, Ho HC, et al. Medical genetics: a marker for stevens-johnson syndrome. Nature (2004) 428:486. doi: $10.1038 / 428486 a$

5. Hung SI, Chung WH, Liu ZS, Chen CH, Hsih MS, Hui RC, et al. Common risk allele in aromatic antiepileptic-drug induced Stevens-Johnson syndrome and toxic epidermal necrolysis in Han Chinese. Pharmacogenomics (2010) 11:349-56. doi: 10.2217/pgs.09.162

6. Wang Q, Zhou JQ, Zhou LM, Chen ZY, Fang ZY, Chen SD, et al. Association between HLA-B*1502 allele and carbamazepine-induced severe cutaneous adverse reactions in Han people of southern China mainland. Seizure (2011) 20:446-8. doi: 10.1016/j.seizure.2011.02.003

7. Wu XT, Hu FY, An DM, Yan B, Jiang X, Kwan P, et al. Association between carbamazepine-induced cutaneous adverse drug reactions and the HLAB*1502 allele among patients in central China. Epilepsy Behav. (2010) 19:4058. doi: 10.1016/j.yebeh.2010.08.007

8. Zhang Y, Wang J, Zhao LM, Peng W, Shen GQ, Xue L, et al. Strong association between HLA-B*1502 and carbamazepine-induced Stevens-Johnson syndrome and toxic epidermal necrolysis in mainland Han Chinese patients. Eur J Clin Pharmacol. (2011) 67:885-7. doi: 10.1007/s00228-011-1009-4

9. Hong W, Chen S, Shao H, Fu Y, Hu Z, Xu A. HLA class I polymorphism in mongolian and hui ethnic groups from Northern China. Hum Immunol. (2007) 68:440-8. doi: 10.1016/j.humimm.2007.01.020

10. Kim RS. A new comparison of nested case-control and casecohort designs and methods. Eur J Epidemiol. (2015) 30:197-207. doi: 10.1007/s10654-014-9974-4

11. Zaccara G, Franciotta D, Perucca E. Idiosyncratic adverse reactions to antiepileptic drugs. Epilepsia (2007) 48:1223-44. doi: 10.1111/j.1528-1167.2007.01041.x

12. Wang $X Q$, Shi $X B, A u ~ R$, Chen FS, Wang F, Lang SY. Influence of chemical structure on skin reactions induced by antiepileptic drugs-The role of the aromatic ring. Epilepsy Res. (2011) 94:213-7. doi: 10.1016/j.eplepsyres.2011.02.005

\section{AUTHOR CONTRIBUTIONS}

QZ conceived this study. XW, LC, XL, and XX recruited patient samples and collected clinical data. Beijing Boao Crystal Biotechnology Company Completed HLA Genotyping. XW provided statistical analyses of the patient data and laboratory analyses.

\section{ACKNOWLEDGMENTS}

This study was funded by CAAE-UCB Scientific Research Grant (Grant No. 2016001), and Key Research Project of the Chinese Ministry of Science and Technology (Grant No. 2016YFC0904400).

13. Man CB, Kwan P, Baum L, Yu E, Lau KM, Cheng AS, et al. Association between HLA-B*1502 allele and antiepileptic drug-induced cutaneous reactions in Han Chinese. Epilepsia (2007) 48:1015-8. doi: 10.1111/j.1528-1167.2007.01022.x

14. Locharernkul C, Loplumlert J, Limotai C, Korkij W, Desudchit T, Tongkobpetch $\mathrm{S}$, et al. Carbamazepine and phenytoin induced StevensJohnson syndrome is associated with HLA-B*1502 allele in Thai population. Epilepsia (2008) 49:2087-91. doi: 10.1111/j.1528-1167.2008.01719.x

15. Chang CC, Too CL, Murad S, Hussein SH. Association of HLA-B*1502 allele with carbamazepine-induced toxic epidermal necrolysis and Stevens-Johnson syndrome in the multi-ethnic Malaysian population. Int J Dermatol. (2011) 50:221-4. doi: 10.1111/j.1365-4632.2010.04745.x

16. Mehta TY, Prajapati LM, Mittal B, Joshi CG, Sheth JJ, Patel DB, et al. Association of HLA-B*1502 allele and carbamazepine-induced StevensJohnson syndrome among Indians. Indian J Dermatol Venereol Leprol. (2009) 75:579-82. doi: 10.4103/0378-6323.57718

17. Kashiwagi M, Aihara M, Takahashi Y, Yamazaki E, Yamane Y, Song Y, et al. Human leukocyte antigen genotypes in carbamazepine-induced severe cutaneous adverse drug response in Japanese patients. J Dermatol. (2008) 35:683-5. doi: 10.1111/j.1346-8138.2008.00548.x

18. Kim SH, Lee KW, Song WJ, Kim SH, Jee YK, Lee SM, et al. Adverse drug reaction research group in Korea. Carbamazepine-induced severe cutaneous adverse reactions and HLA genotypes in Koreans. Epilepsy Res. (2011) 97:1907. doi: 10.1016/j.eplepsyres.2011.08.010

19. McCormack M, Alfirevic A, Bourgeois S, Farrell JJ, Kasperavičiute $\mathrm{D}$, Carrington $\mathrm{M}$, et al. HLA-A*3101 and carbamazepine-induced hypersensitivity reactions in Europeans. N Engl J Med. (2011) 364:1134-43. doi: 10.1056/NEJMoa1013297

20. Amstutz U, Ross CJ, Castro-Pastrana LI, Rieder MJ, Shear NH, Hayden MR, et al. HLA-A 31:01 and HLA-B 15:02 as genetic markers for carbamazepine hypersensitivity in children. Clin Pharmacol Ther. (2013) 94:142-9. doi: 10.1038/clpt.2013.55

Conflict of Interest Statement: The authors declare that the research was conducted in the absence of any commercial or financial relationships that could be construed as a potential conflict of interest.

Copyright (c) 2019 Wang, Chao, Liu, Xu and Zhang. This is an open-access article distributed under the terms of the Creative Commons Attribution License (CC BY). The use, distribution or reproduction in other forums is permitted, provided the original author(s) and the copyright owner(s) are credited and that the original publication in this journal is cited, in accordance with accepted academic practice. No use, distribution or reproduction is permitted which does not comply with these terms. 